\title{
Erratum
}

Dans I'article intitulé : "Spécificités de la chimiothérapie chez le sujet âgé " paru dans la revue Oncologie, volume 9, numéro 11, novembre 2007, il manquait un auteur :

I. Debrix

Pharmacie, hôpital Tenon, Assistance publique-Hôpitaux de Paris, 4, rue de la Chine, F-75020 Paris, France

II fallait donc lire sur la couverture et dans le sommaire :

D. Avenin, F. Selle, J. Gligorov, A. Khalil, E. Bouvard, M. Japkowicz, I. Debrix, J.-P. Lotz Spécificités de la chimiothérapie chez le sujet âgé

Et en page 783 :

\section{Spécificités de la chimiothérapie chez le sujet âgé}

D. Avenin ${ }^{1}$, F. Selle ${ }^{1}$, J. Gligorov ${ }^{1}$, A. Khalil ${ }^{1}$, E. Bouvard² ${ }^{2}$ M. Japkowicz ${ }^{1}$, I. Debrix ${ }^{3}$, J.-P. Lotz ${ }^{1}$

${ }^{1}$ Service d'oncologie médicale, hôpital Tenon, Cancer-Est, Assistance publique-Hôpitaux de Paris, université Pierre-et-Marie-Curie, 4, rue de la Chine, F-75020 Paris, France

${ }^{2}$ Service de médecine interne, hôpital Tenon, Assistance publique-Hôpitaux de Paris, université Pierre-et-Marie-Curie, F-75020 Paris, France

${ }^{3}$ Pharmacie, hôpital Tenon, Assistance publique-Hôpitaux de Paris, 4, rue de la Chine,

F-75020 Paris, France

Correspondance : jean-pierre.lotz@tnn.aphp.fr 